\title{
Square functions of Calderón type and applications
}

Steve Hofmann and John L. Lewis

\begin{abstract}
We establish $L^{2}$ and $L^{p}$ bounds for a class of square functions which arises in the study of singular integrals and boundary value problems in non-smooth domains. As an application, we present a simplified treatment of a class of parabolic smoothing operators which includes the caloric single layer potential on the boundary of certain minimally smooth, non-cylindrical domains.
\end{abstract}

\section{Introduction and notation.}

In this note we prove certain square function estimates which are in the spirit of those considered by David, Journé, and Semmes [DJS, Section 11]. In particular, they (essentially) include square function estimates for solutions of the heat equation in time varying domains [HL, Theorem 3.1], but our treatment here is of a purely real variable and geometric nature, and does not depend on properties of solutions of a PDE. Our approach will be based on an idea of P. Jones [JnsP], who gave a proof of the deep result of Coifman, McIntosh, and Meyer [CMM] concerning the $L^{2}$ boundedness of the Cauchy integral operator along a Lipschitz curve, by viewing the Lipschitz curve as (locally) a pertubation of an approximating line, and then controlling the resulting error terms by a certain Carleson measure estimate. In this context see also the work of Fang [Fng], and the monograph of Christ [Ch]. We note that an important antecedent of Jones' ideas is contained in the work of Dorronsoro [Do]. We shall apply our square function estimates 
to obtain an alternative proof of [H2, Theorem 3], which is a regularity result for a class of parabolic smoothing operators which includes the caloric single layer on the boundary of certain non-smooth time-varying domains.

Our main application being parabolic, we shall state and prove a parabolic version of our square function estimates. The elliptic version is similar, but a bit simpler. Indeed, another application of our method has been given by D. Mitrea, M. Mitrea, and M. Taylor [MMT, Section 1], who follow our approach here to prove certain square function estimates that are useful in their work on elliptic boundary value problems in non-smooth Riemannian manifolds.

Let us now introduce some notation. Our operators are modeled on operators arising from the theory of layer potential on non-smooth, time-varying domains. The class of domains under consideration have boundaries given (at least locally) as graphs of functions $A(x, t), x \in$ $\mathbb{R}^{n-1}, t \in \mathbb{R}$, which are Lipschitz in space, uniformly in time, and which satisfy a certain half order smoothness condition in the time variable, which is related to the BMO Sobolev spaces of Strichartz [Stz]. To be more precise, we suppose that there exists a constant $\beta$ such that

$$
|A(x, t)-A(y, t)| \leq \beta|x-y|,
$$

and

$$
\left\|\mathbb{D}_{n} A\right\|_{*} \leq \beta
$$

Here, $\|\cdot\|_{*}$ denotes the parabolic BMO norm (defined below), and, following Fabes and Riviere [FR], we have defined a half-order time derivative by

$$
\mathbb{D}_{n} A(x, t)=\left(\frac{\tau}{\|(\xi, \tau)\|} \widehat{A}(\xi, \tau)\right)^{\vee}(x, t),
$$

where $\widehat{ }$ and $\vee$ denote respectively the Fourier and inverse Fourier transforms on $\mathbb{R}^{n}$, and $\xi, \tau$ denote, respectively, the space and time variables on the Fourier transform side. Also, $\|z\|$ denotes the parabolic "norm" of $z$. We recall that this "norm" satisfies the non-isotropic dilation invariance property $\left\|\left(\delta x, \delta^{2} t\right)\right\| \equiv \delta\|(x, t)\|$. Indeed, $\|(x, t)\|$ is defined as the unique positive solution $\rho$ of the equation

$$
\sum_{i=1}^{n-1} \frac{x_{i}^{2}}{\rho^{2}}+\frac{t^{2}}{\rho^{4}}=1
$$


We note that the class of functions $A(x, t)$ satisfying (1.1) and (1.2), has been introduced (with a somewhat different, albeit equivalent formulation) in [LM], and considered further in [H1], [H2], and [HL]. In particular, it is shown in [H1] that this class of functions is the natural sharp parabolic analogue, of the class of Lipschitz functions in the elliptic theory, for the development of a Calderón type singular integral theory [Ca1], [Ca2]. Indeed, in [H1] it is shown that

$$
\left\|\left(\sqrt{\Delta-\frac{\partial}{\partial t}}, A\right)\right\|_{\mathrm{op}} \approx\left\|\nabla_{x} A\right\|_{\infty}+\left\|\mathbb{D}_{n} A\right\|_{*},
$$

where $\approx$ means the two quantities are bounded by constant multiples of each other. Moreover, $\|\cdot\|_{\text {op }}$ denotes the operator norm on $L^{2}\left(\mathbb{R}^{n-1}\right)$, and

$$
\nabla_{x} \equiv\left(\frac{\partial}{\partial x_{1}}, \ldots, \frac{\partial}{\partial x_{n-1}}\right)
$$

Since $(\sqrt{\Delta-\partial / \partial t}, A)$ is the parabolic version of the first Calderón commutator, we define the "commutator" norm of $A$ by

$$
\|A\|_{\text {comm }} \equiv\left\|\nabla_{x} A\right\|_{\infty}+\left\|\mathbb{D}_{n} A\right\|_{*} .
$$

Of course, (1.1) and (1.2) say that this quantity is finite. In [H1] it is also shown that finiteness of (1.6) implies the parabolic Lipschitz condition

$$
|A(x, t)-A(y, s)| \leq c \beta\|(x, t)-(y, s)\| \approx c \beta\left(|x-y|+|t-s|^{1 / 2}\right) .
$$

We recall now that parabolic BMO is the space of all locally integrable functions modulo constants satisfying

$$
\|b\|_{*} \equiv \sup _{B} \frac{1}{|B|} \int_{B}\left|b(z)-m_{B} b\right| d z<\infty .
$$

Here, $z=(x, t)$ and $B$ denotes the parabolic ball

$$
B \equiv B_{r}\left(z_{0}\right) \equiv\left\{z \in \mathbb{R}^{n}:\left\|z-z_{0}\right\|<r\right\},
$$

where $|B|$ denotes the Lesbegue $n$ measure of $B$ and

$$
m_{B} b \equiv \frac{1}{|B|} \int_{B} b(z) d z
$$


We note that $\left|B_{r}\left(z_{0}\right)\right| \equiv c r^{d}$ where $c$ is a constant and $d=n+1$ is the homogeneous dimension of $\mathbb{R}^{n}$ endowed with the metric induced by $\|\cdot\|$, as defined in (1.4). We observe that $\mathbb{R}^{n}$ so endowed is a space of homogeneous type in the sense of Coifman and Weiss [CW]. Indeed, there is a polar decomposition

$$
\begin{aligned}
& z \equiv(x, t) \equiv\left(\rho \theta_{1}, \ldots, \rho \theta_{n-1}, \rho^{2} \theta_{n}\right), \\
& d z \equiv d x d t \equiv \rho^{d-1}\left(1+\theta_{n}^{2}\right) d \rho d \theta
\end{aligned}
$$

where $\theta=\left(\theta_{1}, \ldots, \theta_{n}\right),|\theta|=1$, and $d \theta$ denotes surface area on the unit sphere.

Finally, we note that througout the sequel, we shall use the convenient notation

$$
z=(x, t) \in \mathbb{R}^{n}, \quad v=(y, s) \in \mathbb{R}^{n},
$$

and we shall denote the parabolic dilations by the convenient notation

$$
\delta^{\alpha} z \equiv\left(\delta x, \delta^{2} t\right)
$$

where $\alpha$ will always denote the n-dimensional multi-index $(1, \ldots, 1,2)$.

In the next section, we introduce the class of operators which we shall consider, and state our results.

\section{Statement of results.}

We begin by defining our square functions. To this end, let $H \in$ $C^{1}\left(\mathbb{R}^{n} \backslash\{0\}\right)$ satisfy the homogeneity condition

$$
H\left(\delta^{\alpha} z\right)=\delta^{-d-1} H(z), \quad \text { for } z=(x, t), d=n+1,
$$

and assume that $F \in C^{1}(\mathbb{R})$ with

$$
\begin{aligned}
& |F(r)| \leq c_{F} \frac{1}{1+|r|^{d+1}}, \\
& \left|F^{\prime}(r)\right| \leq c_{F} \frac{1}{1+|r|^{d+2}},
\end{aligned}
$$


whenever $r \in \mathbb{R}$. For $F, H$ as above define a square function $G$ of "Calderón type" by setting

$$
\begin{gathered}
R_{\lambda} f(z) \equiv \lambda \int_{\mathbb{R}^{n}} H(z-v) F\left(\frac{A(z)-A(v)+\lambda}{\|z-v\|}\right) f(v) d v \\
G f(z)=\left(\int_{0}^{\infty}\left|R_{\lambda} f(z)\right|^{2} \frac{d \lambda}{\lambda}\right)^{1 / 2} .
\end{gathered}
$$

Let $\omega$ be a parabolic $A_{2}$ weight (these are defined in the usual way, in this case with respect to parabolic balls, or cubes), and $f \in L_{\omega}^{2}\left(\mathbb{R}^{n}\right)$. As usual,

$$
\|f\|_{2, \omega} \equiv\left(\int|f(x)|^{2} \omega(x) d x\right)^{1 / 2} .
$$

We shall work with weighted $L^{2}$, because, when dealing with square functions, this is a particularly suitable way to obtain $L^{p}$ bounds (via extrapolation - see $[\mathrm{GR}]$ ). Furthermore our main application is to rough singular integral operators which do not satisy the standard CalderónZygmund kernel estimates, and thus cannot be shown to be bounded on $L^{p}$ via the standard program. However, as usual, it is really our unweighted $L^{2}$ bounds which are the heart of the matter - the extension to the weighted case is routine. We shall prove the following theorem.

Theorem 2.5. Suppose that for $H, F$ as above (see (2.1) and (2.2)) we have either $F$ is odd and $H(x, t)$ is odd in $x$ for each fixed $t$; or else that $F$ is even, $H(x, t)$ is even in $x$ for each fixed $t$, and also that $\int_{\mathbb{R}} F(r) d r=0$. If $\|A\|_{\text {comm }} \leq \beta<\infty$, and $\omega \in A_{2}$, then there exists a positive integer $N$ depending only on $d$ such that

$$
\|G f\|_{2, \omega} \leq c_{F, H, \omega}(1+\beta)^{N}\|f\|_{2, \omega} \text {. }
$$

REMARK. Here and in the sequel, when we indicate that a constant depends on $\omega$, we mean that it actually depends only on the $A_{2}$ constant of $\omega$, so that $L^{p}$ bounds follow by extrapolation [GR].

Theorem 2.5 is easily generalized, in a way that is useful for some applications. Indeed, let $H, F$, be as in $(2.1),(2.2)$, and let $B: \mathbb{R}^{n} \longrightarrow \mathbb{R}$ with

$$
\|B\|_{\text {comm }} \leq \beta_{0}<\infty
$$


Let $A$ be as in Theorem 2.5 and put

$$
\begin{aligned}
\widetilde{R}_{\lambda} f(z) \equiv \lambda \int_{\mathbb{R}^{n}} H(z-v) \frac{B(z)-B(v)}{\|z-v\|} \\
\cdot F\left(\frac{A(z)-A(v)+\lambda}{\|z-v\|}\right) f(v) d v, \\
\widetilde{G} f(z)=\left(\int_{0}^{\infty}\left|\widetilde{R}_{\lambda} f(z)\right|^{2} \frac{d \lambda}{\lambda}\right)^{1 / 2} .
\end{aligned}
$$

We then have

Theorem 2.8. Let $H, F$, and $A$ be as in Theorem 2.5, and let $B$ satisfy $\|B\|_{\text {comm }} \leq \beta_{0}<\infty$. Suppose that either $F$ is odd and $H(x, t)$ is even in $x$ for each fixed $t$; or else that $F$ is even, $H(x, t)$ is odd in $x$ for each fixed $t$, and also that $\int_{\mathbb{R}} F(r) d r=0$. If $\omega \in A_{2}$, then there exists a positive integer $N$ depending only on $d$ such that

$$
\|\widetilde{G} f\|_{2, \omega} \leq c_{F, H, \omega} \beta_{0}(1+\beta)^{N}\|f\|_{2, \omega} .
$$

In our applications the square functions defined in (2.3)-(2.4) and (2.6)-(2.7) model the second derivatives of the single layer potential mapped to $\mathbb{R}_{+}^{n+1}$. We shall also describe here a model for higher order derivatives. We refrain from stating the most general result of this type as it would lead us too far astray from the purposes of this paper. Suppose $L \in C^{1}\left(\mathbb{R}^{n} \backslash\{0\}\right)$ with

$$
L\left(\delta^{\alpha} z\right)=\delta^{-d-2} L(z), \quad z \in \mathbb{R}^{n},
$$

and let $E \in C^{1}(\mathbb{R})$ with

$$
\begin{aligned}
& |E(r)| \leq c_{E} \frac{1}{1+|r|^{d+2}}, \\
& \left|E^{\prime}(r)\right| \leq c_{E} \frac{1}{1+|r|^{d+3}},
\end{aligned}
$$

whenever $r \in \mathbb{R}$. Suppose that either $E$ is even with $\int_{\mathbb{R}} E(r) d r=0$ and $L(x, t)$ is odd in $x$ for each fixed $t$; or else that $E$ is odd, with $\int_{\mathbb{R}} r E(r) d r=0$, and $L(x, t)$ is even in $x$ for each fixed $t$. Next assume 
that $\widetilde{L} \in C^{1}\left(\mathbb{R}^{n} \backslash\{0\}\right)$ satisfies (2.9) and $\widetilde{E} \in C^{1}(\mathbb{R})$ satisfies (2.10). Suppose that either $\widetilde{E}$ is even with $\int_{\mathbb{R}} \widetilde{E}(r) d r=0$ while $\widetilde{L}(x, t)$ is even in $x$ for each fixed $t$; or else that $\widetilde{E}$ is odd with $\int_{\mathbb{R}} r \widetilde{E}(r) d r=0$, while $\widetilde{L}(x, t)$ is odd in $x$ for each fixed $t$. We set

$$
\begin{gathered}
T_{\lambda} f(z) \equiv \lambda^{2} \int_{\mathbb{R}^{n}} L(z-v) E\left(\frac{A(z)-A(v)+\lambda}{\|z-v\|}\right) f(v) d v, \\
\widetilde{T}_{\lambda} f(z) \equiv \lambda^{2} \int_{\mathbb{R}^{n}} \widetilde{L}(z-v) \frac{B(z)-B(v)}{\|z-v\|} \\
\cdot \widetilde{E}\left(\frac{A(z)-A(v)+\lambda}{\|z-v\|}\right) f(v) d v,
\end{gathered}
$$

where $\|A\|_{\text {comm }} \leq \beta<\infty,\|B\|_{\text {comm }} \leq \beta_{0}<\infty$, and

$$
\begin{aligned}
& g(f)(z)=\left(\int_{0}^{\infty}\left|T_{\lambda} f(z)\right|^{2} \frac{d \lambda}{\lambda}\right)^{1 / 2} \\
& \widetilde{g}(f)(z)=\left(\int_{0}^{\infty}\left|\widetilde{T}_{\lambda} f(z)\right|^{2} \frac{d \lambda}{\lambda}\right)^{1 / 2} .
\end{aligned}
$$

With this notation we have

Theorem 2.13. Let $E, L, \widetilde{E}, \widetilde{L}, g, \widetilde{g}, A, B$, be as above. Then there exists a positive integer $N=N(d)$ such that if $f, \omega$ are as in Theorem 2.5, we have

$$
\|g(f)\|_{2, \omega}+\beta_{0}^{-1}\|\widetilde{g}(f)\|_{2, \omega} \leq c(1+\beta)^{N}\|f\|_{2, \omega} .
$$

where $c$ depends on $\omega, E, L, \widetilde{E}, \widetilde{L}$, and $d$.

We shall not bother to give the proof of Theorem 2.13 in this note, as the interested reader could easily supply it after reading the proofs of Theorems 2.5 and 2.8 .

To conclude this section, we now describe the parabolic smoothing operators which are our main application. Let $J$ denote a kernel which satisfies the homogeneity property

$$
J\left(\delta^{\alpha} z\right) \equiv \delta^{-d+1} J(z),
$$

where $d=n+1$ and $z \in \mathbb{R}^{n}$. We also assume that $J$ is sufficiently smooth away from the origin, i.e., $J \in C^{m}\left(\mathbb{R}^{n} \backslash\{0\}\right)$, for some large 
$m$. With this notation, let $E$ denote either sine or cosine, and define "smoothing operators of Calderón type" by

$$
\begin{gathered}
S_{A} f(z) \equiv \int_{\mathbb{R}^{n}} J(z-v) E\left(\frac{A(z)-A(v)}{\|z-v\|}\right) f(v) d v, \\
U_{A, B} f(z) \equiv \int_{\mathbb{R}^{n}} J(z-v) E\left(\frac{A(z)-A(v)}{\|z-v\|}\right) \\
\cdot \frac{B(z)-B(v)}{\|z-v\|} f(v) d v .
\end{gathered}
$$

We shall give a simpler proof of the following result of the first author [H2, Theorem 3]. Let $L_{1,1 / 2}^{p}$ denote the parabolic Sobolev space defined as the collection of all $\mathrm{f}$ having a spatial gradient and $1 / 2$ a time derivative in $L^{p}$, i.e., those $f$ for whom the following norm is finite

$$
\|f\|_{L_{1,1 / 2}^{p}} \equiv\left\|\nabla_{x} f\right\|_{p}+\left\|\mathbb{D}_{n} f\right\|_{p}
$$

Theorem 2.16. Let $\|A\|_{\mathrm{comm}},\|B\|_{\mathrm{comm}}<\infty$ and $f \in L^{p}\left(\mathbb{R}^{n}\right), 1<$ $p<\infty$. Suppose that $J$ is sufficiently smooth away from the origin. If $J(x, t)$ has the same parity in $x$ as does $E$, then for some large positive $N$, we have

$$
\left\|S_{A} f\right\|_{L_{1,1 / 2}^{p}} \leq c_{p, J}\left(1+\|A\|_{\text {comm }}\right)^{N}\|f\|_{p} .
$$

Similarly if $J(x, t)$ has opposite parity in $x$ to that of $E$, then

$$
\left\|U_{A, B} f\right\|_{L_{1,1 / 2}^{p}} \leq c_{p, J}\|B\|_{\text {comm }}\left(1+\|A\|_{\text {comm }}\right)^{N}\|f\|_{p} .
$$

REMARKS. 1) Using the method of [CDM], one can immediately replace the trigonometric function $E$ by any sufficiently smooth function defined on $\mathbb{R}$ with the same parity as $E$. One can also treat layer potentials via this method.

2) Theorem 3 in [H2] is stated for $A_{2}$ weights but implies our Theorem 2.16 by extrapolation.

In the next section (3), we treat our square functions (theorems 2.5 and 2.8). In the last section (4), we give the alternative proof of Theorem 2.16. 


\section{Proofs of theorems 2.5, 2.8.}

We begin with a simple lemma. For $(\lambda, z),(\lambda, v) \in \mathbb{R}_{+}^{n+1}$, let $K_{\lambda}(z, v)$ be a family of real valued kernels satisfying

$$
\begin{gathered}
\left|K_{\lambda}(z, v)\right| \leq c_{K} \frac{\lambda}{(\lambda+\|z-v\|)^{d+1}}, \\
\left|K_{\lambda}(z, v)-K_{\lambda}(z, \widetilde{v})\right| \\
\leq c_{K}\|v-\widetilde{v}\| \min \left\{\frac{1}{\lambda^{d}\|z-v\|}, \frac{\lambda}{\|z-v\|^{d+2}}\right\},
\end{gathered}
$$

whenever $2\|v-\widetilde{v}\| \leq\|z-v\|$. Let $\omega$ be a parabolic $A_{2}$ weight. Put

$$
K_{\lambda} f(z)=\int_{\mathbb{R}^{n}} K_{\lambda}(z, v) f(v) d v, \quad z \in \mathbb{R}^{n} .
$$

The following result is standard, and we omit the proof.

Lemma 3.3. Let $\left(K_{\lambda}\right)$ satisfy (3.1), (3.2) and let $\omega, f$ be as above. If $K_{\lambda} 1 \equiv 0$ for each $\lambda>0$, then

$$
\int_{\mathbb{R}_{+}^{n+1}}\left(K_{\lambda} f\right)^{2}(z) \omega(z) \frac{d z d \lambda}{\lambda} \leq c_{K, \omega}\|f\|_{2, \omega}^{2} .
$$

In Lemma 3.3, $c_{K, \omega}$ denotes a constant depending only on $K, d$, and the $A_{2}$ constant of $\omega$, which is the same convention we used in Section 2. Lemma 3.3 is stated in [Ch, p. 69, Theorem 20] for $\omega=1$ (see also [CJ]) under slightly weaker hypotheses.

Proof of Theorem 2.5. Let $P \in C_{0}^{\infty}\left(B_{1}(0)\right)$ be an even function with $\int_{\mathbb{R}^{n}} P_{\lambda}(z) d z \equiv 1$, where as usual $P_{\lambda}(z) \equiv \lambda^{-d} P\left(\lambda^{-\alpha} z\right)$ and let $f \longrightarrow P_{\lambda} f$ be the convolution operator whose kernel is $P_{\lambda}(z)$. Put

$$
Q_{\lambda}^{*} f(z) \equiv \lambda \int_{\mathbb{R}^{n}} H(z-v) F\left(\frac{\left\langle\nabla_{z^{\prime}} P_{\lambda} A(z), z^{\prime}-v^{\prime}\right\rangle+\lambda}{\|z-v\|}\right) f(v) d v,
$$

where $z^{\prime}=x, v^{\prime}=y$ if $z=(x, t)$ and $v=(y, s)$. Then

$$
\begin{aligned}
G f(z) & \leq\left(\int_{0}^{\infty}\left|\left(R_{\lambda}-Q_{\lambda}^{*}\right) f(z)\right|^{2} \frac{d \lambda}{\lambda}\right)^{1 / 2}+\left(\int_{0}^{\infty}\left|Q_{\lambda}^{*} f(z)\right|^{2} \frac{d \lambda}{\lambda}\right)^{1 / 2} \\
& =G_{1} f(z)+G_{2} f(z) .
\end{aligned}
$$


We set $V_{\lambda} \equiv R_{\lambda}-Q_{\lambda}^{*}$ and observe from (2.1) and (2.2) that the kernel $V_{\lambda}(z, v)$ of $V_{\lambda}$ satisfies

$$
\begin{aligned}
\left|V_{\lambda}(z, v)\right| \leq & c(1+\beta)^{d+2} \frac{\lambda}{(\lambda+\|z-v\|)^{d+2}} \\
& \cdot\left|A(z)-A(v)-\left\langle\nabla_{z^{\prime}} P_{\lambda} A(z), z^{\prime}-v^{\prime}\right\rangle\right|,
\end{aligned}
$$

where $c$ depends on $F, H, d$. Using (3.5) and (1.7) we deduce that $V_{\lambda}$ satisfies (3.1) with $K$ replaced by $V$ and $c_{K}$ replaced by $c(1+\beta)^{d+3}$. Also by the same argument we see that the kernel of $Q_{\lambda}^{*}$ satisfies (3.1) with $Q^{*}=K$ and the same constants as $V$. Moreover, since $H \in$ $C^{1}\left(\mathbb{R}^{n} \backslash\{0\}\right)$ we find in addition from $(2.1),(2.2)$ and (1.7), that the kernels of $V_{\lambda}, Q_{\lambda}^{*}$ satisfy (3.2) with the same constants as in (3.1).

First we consider $G_{1}$ in (3.4). This term will be treated using the main idea in $[\mathrm{JnsP}]$, but with the particular details closer to the exposition in [Ch]. From the above discussion we see that we may follow the standard approach, as in [CM], to handle $K_{\lambda}=V_{\lambda}-\left(V_{\lambda} 1\right) P_{\lambda}$, via Lemma 3.3 since $K_{\lambda} 1 \equiv 0$ for each $\lambda>0$. Thus to show

$$
\left\|G_{1} f\right\|_{2, \omega} \leq c_{F, H, \omega}(1+\beta)^{N}\|f\|_{2, \omega}
$$

we need only prove

$$
\int_{0}^{\infty} \int_{\mathbb{R}^{n}}\left(V_{\lambda} 1 P_{\lambda} f\right)^{2} \omega \frac{d z d \lambda}{\lambda} \leq c_{F, H, \omega}(1+\beta)^{2 N}\|f\|_{2, \omega},
$$

i.e., that

$$
d \nu(\lambda, z)=\left(V_{\lambda} 1(z)\right)^{2} \omega(z) \frac{d z d \lambda}{\lambda}
$$

is a weighted Carleson measure with norm comparable to the constants in Theorem 2.5. To this end let $z_{0} \in \mathbb{R}^{n}, r>0$, and let $\chi, \chi^{*}$ denote the characteristic functions of $B_{10 r}\left(z_{0}\right), \mathbb{R}^{n} \backslash B_{10 r}\left(z_{0}\right)$, respectively. Fixing this ball, and using (3.1) for $V_{\lambda}$ we deduce, as usual, that it suffices to replace 1 by $\chi$ in (3.7). Next we put $\widetilde{A}(z)=\psi\left(\left\|z-z_{0}\right\|\right)\left(A(z)-A\left(z_{0}\right)\right)$, $z \in \mathbb{R}^{n}$, where $\psi \in C_{0}^{\infty}(-20 r, 20 r)$ is an even function with $\psi \equiv 1$ on $[-15 r, 15 r]$. Then $V_{\lambda} \chi(z)$ is unchanged for $z \in B_{10 r}\left(z_{0}\right), 0<\lambda<r$, if we replace $A$ in its definition by $\widetilde{A}$. Moreover from [H2, Section 6 , Lemma 2] we have

i) $\|\widetilde{A}\|_{\mathrm{comm}} \leq c\|A\|_{\mathrm{comm}}$.

ii) For $1<p<\infty,\|\mathbb{D} \widetilde{A}\|_{p}^{p} \leq c_{p} \beta^{p} r^{d}$, 
where the parabolic fractional derivative operator $\mathbb{D}$ is defined by the Fourier multiplier

$$
\widehat{\mathbb{D f}} \equiv\|\zeta\| \widehat{f} .
$$

Using (3.5), Schwarz's inequality, and the change of variable $\lambda \longrightarrow \lambda / 2^{l}$ we obtain, for $N$ large enough that

$$
\begin{gathered}
(1+\beta)^{-2 N} \int_{0}^{r} \int_{B_{r}\left(z_{0}\right)}\left(V_{\lambda} \chi\right)^{2}(z) \omega(z) \frac{d z d \lambda}{\lambda} \\
\leq c \sum_{l=0}^{\infty} 2^{-l} \int_{0}^{\infty} \int_{\mathbb{R}^{n}} \lambda^{-d-2}\left(\int_{B_{\lambda}(z)} \mid \widetilde{A}(z)-\widetilde{A}(v)\right. \\
\left.(3.9) \quad-\left.\left\langle\nabla_{z^{\prime}} P_{2^{-l} \lambda} \widetilde{A}(z), z^{\prime}-v^{\prime}\right\rangle\right|^{2} d v\right) \\
\cdot \omega(z) \frac{d z d \lambda}{\lambda} \\
\leq c_{\omega} \beta^{2} \omega\left(B_{r}\left(z_{0}\right)\right),
\end{gathered}
$$

where the last inequality follows from (3.8) and an argument involving Plancherel's Theorem in the case $\omega \equiv 1$ (see [H2, Section 5] for more details) or else the argument of [H2, Section 6, Lemma 3] in the weighted case. Thus (3.6) holds.

To prove the analogue of (3.6) with $G_{1}$ replaced by $G_{2}$ we note that (3.1), (3.2) for $Q_{\lambda}^{*}$, and Lemma 3.3 imply that it is enough to show that $Q_{\lambda}^{*} 1 \equiv 0$. To do this we introduce the parabolic polar coordinates defined in (1.10) to get

$$
Q_{\lambda}^{*} 1(z)=\lambda \int_{S}\left(\int_{0}^{\infty} F\left(\left\langle\vec{a}, \sigma^{\prime}\right\rangle+\frac{\lambda}{\rho}\right) \frac{d \rho}{\rho^{2}}\right) H(\sigma) \Phi(\sigma) d \sigma
$$

where $\vec{a}=\nabla_{z^{\prime}} P_{\lambda} A(z), \Phi(\sigma)=\left(1+\sigma_{n}^{2}\right)$, and $\sigma=\left(\sigma^{\prime}, \sigma_{n}\right) \in S=$ the unit sphere in $\mathbb{R}^{n}$. We change variables in the above integral by $\rho \longrightarrow \lambda \rho$, then $r=1 / \rho$, then $r \longrightarrow r-\left\langle\vec{a}, \sigma^{\prime}\right\rangle$, to obtain

$$
Q_{\lambda}^{*} 1(z)=\int_{S}\left(\int_{\left\langle\vec{a}, \sigma^{\prime}\right\rangle}^{\infty} F(r) d r\right) H(\sigma) \Phi(\sigma) d \sigma=0,
$$

since our hypotheses in Theorem 2.5 guarantee that this last expression is zero. Indeed $\int_{\left\langle\vec{a}, \sigma^{\prime}\right\rangle}^{\infty} F(r) d r$ is a function of $\sigma^{\prime}$ having opposite parity to $H(\sigma) \Phi(\sigma)$, for each fixed non-zero $\vec{a}$. The case $\vec{a}=0$ is much simpler: 
if $H$ is odd in $\sigma^{\prime}$, then clearly $\int_{S} H(\sigma) \Phi(\sigma) d \sigma=0$, and if $F$ is even with $\int_{-\infty}^{\infty} F(r) d r=0$, then $\int_{0}^{\infty} F(r) d r=0$. Thus (3.6) holds also for $G_{2}$, and the conclusion of Theorem 2.5 follows.

Proof of Theorem 2.8. We shall be brief, since the ideas are now familiar. Put

$$
\begin{aligned}
& U_{\lambda} f(z) \\
\equiv & \lambda \int_{\mathbb{R}^{n}} H(z-v) \frac{\left\langle\nabla_{z^{\prime}} P_{\lambda} B(z), z^{\prime}-v^{\prime}\right\rangle}{\|z-v\|} F\left(\frac{A(z)-A(v)+\lambda}{\|z-v\|}\right) f(v) d v .
\end{aligned}
$$

Then as in (3.4)

$$
\begin{aligned}
\widetilde{G} f(z) & \leq\left(\int_{0}^{\infty}\left|\left(\widetilde{R}_{\lambda}-U_{\lambda}\right) f(z)\right|^{2} \frac{d \lambda}{\lambda}\right)^{1 / 2}+\left(\int_{0}^{\infty}\left|U_{\lambda} f(z)\right|^{2} \frac{d \lambda}{\lambda}\right)^{1 / 2} \\
& =\widetilde{G}_{1} f(z)+\widetilde{G}_{2} f(z) .
\end{aligned}
$$

If $\widetilde{V}_{\lambda}=\widetilde{R}_{\lambda}-U_{\lambda}$, then as in (3.5) we deduce

$$
\begin{aligned}
\left|\widetilde{V}_{\lambda}(z, v)\right| \leq & c(1+\beta)^{d+2} \min \left\{\frac{\lambda}{\|z-v\|^{d+2}}, \frac{1}{\lambda^{d}\|z-v\|}\right\} \\
\cdot & \left|B(z)-B(v)-\left\langle\nabla_{z^{\prime}} P_{\lambda} B(z), z^{\prime}-v^{\prime}\right\rangle\right|
\end{aligned}
$$

where $c$ depends on $F, H, d$. Using this inequality in place of (3.5) we can now repeat the argument following (3.5) through (3.9) to get that (3.6) holds with $G_{1}$ replaced by $\widetilde{G}_{1}$ and constants as in Theorem 2.8 . As for $\widetilde{G}_{2}$ we note from (1.7) that the kernel of $U_{\lambda}$ can be written as a sum of $L^{\infty}$ functions (the components of $\left.\nabla_{z^{\prime}} P_{\lambda} B(z)\right)$ times operators whose kernels satisfy the hypotheses of Theorem 2.5. Thus (3.6) holds with $G_{1}$ replaced by $\widetilde{G}_{2}$ and constants as in Theorem 2.8 , and we are done.

\section{Alternative proof of Theorem 2.16.}

Next we shall use Theorems 2.5, 2.8, and 2.13, to give an alternate proof of Theorem 2.16 (i.e. essentially [H2, Theorem 3]). Our reduction of the proof of Theorem 2.16 to the square function estimates which we have proved in the previous theorems, will be in the spirit of some 
recent work of Li, McIntosh, and Semmes [LiMS, Section 4]. To begin, we consider the operator $S=S_{A}$ of Theorem 2.16. For specificity, we consider

$$
S f(z) \equiv \int_{\mathbb{R}^{n}} J(z-v) \cos \left(\frac{A(z)-A(v)}{\|z-v\|}\right) f(v) d v,
$$

where

a) $J(x, t)$ is even in $x$, for each fixed $t$,

b) $J\left(\lambda^{\alpha} z\right) \equiv \lambda^{1-d} J(z), z \in \mathbb{R}^{n}$,

c) $J \in C_{0}^{N}\left(\mathbb{R}^{n} \backslash\{0\}\right)$, for some large $N$.

Our goal is to show that for some large $N$ and for each $j, 1 \leq j \leq n$, we have

$$
\left\|\mathbb{D}_{j} S f\right\|_{p} \leq c_{J, p}(1+\beta)^{N}\|f\|_{p} .
$$

whenever $f \in L^{p}\left(\mathbb{R}^{n}\right)$, and $1<p<\infty$. Here, $\mathbb{D}_{j}=\partial / \partial x_{j}$ for $1 \leq$ $j \leq n-1$, and $\mathbb{D}_{n}$ is the $1 / 2$ order time derivative defined in Section 1 . Since $\nabla_{x} A \in L^{\infty}\left(\mathbb{R}^{n}\right)$, we have that, modulo pointwise multiplication by a bounded function, each $\mathbb{D}_{j} S, 1 \leq j \leq n-1$, gives rise to a standard parabolic Calderón-Zygmund operator which falls under the scope of [H2, Theorem 1] (to see this, just differentiate formally under the integral sign in the definition of $S f$ - this formal computation may be justified by smoothly truncating the kernel $J$, and obtaining bounds independent of the truncation). Thus it suffices to prove the case $j=n$ of (4.2). In fact if $\omega$ is an $A_{2}$ weight and $f \in L_{\omega}^{2}\left(\mathbb{R}^{n}\right)$, we shall show that

$$
\left\|\mathbb{D}_{n} S f\right\|_{2, \omega} \leq c_{J, \omega}(1+\beta)^{N}\|f\|_{2, \omega}
$$

Once (4.3) is proved, the Theorem then follows from extrapolation (see [GR, Chapter 4, Theorem 5.19]). We remark that the operator $\mathbb{D}_{n} S$ cannot be viewed as a standard Calderón-Zygmund operator (modulo multiplication by a bounded function), and hence does not fall under the scope of [H2, Theorem 1], nor can one use the classical CalderónZygmund theory to pass from $L^{2}$ bounds to $L^{p}$. The failure of the standard C-Z estimates in this case is related to the fact that the chain rule does not hold for fractional derivatives like $\mathbb{D}_{n}$. 
To make our arguments rigorous, we observe that since

$$
|A(z)-A(v)| \leq c\|A\|_{\text {comm }}\|z-v\|
$$

(see (1.7)), we can replace the cosine in the definition of $S f$ by $\Lambda$ where $\Lambda(r)=\phi(r) \cos (r)$ and $\phi \in C_{0}^{\infty}(\mathbb{R})$ is an even function with $\phi \equiv 1$ on $[-c \beta, c \beta]$. Clearly we can also choose $\phi$ so that $\int_{-\infty}^{\infty} \Lambda(r) d r=0$. We make the a priori assumption that $f \in C_{0}^{\infty}\left(\mathbb{R}^{n}\right), A \in C^{\infty}\left(\mathbb{R}^{n}\right)$, and that $J$ has been smoothly truncated so that it is supported on a parabolic annulus. These assumptions allow us easily to justify repeated differentiations and integrations by parts in the argument which follows. In the rest of the proof we shall systematically suppress the truncation, so as not to tire the reader with routine details. This means that we shall be ignoring certain error terms which arise as a result of the truncation, but these are not difficult to handle. Of course, our estimates will not have any quantitative dependence upon our a priori assumptions.

Under these assumptions we first use a construction of Kenig and Stein (which appeared first in a paper of Dahlberg [D]; see also [DKPV] and [HL] for applications related to the present paper), to write $S f(z)=$ $\lim _{\lambda \rightarrow 0} S_{\lambda} f(z)$, where

$$
S_{\lambda} f(z) \equiv \int_{\mathbb{R}^{n}} J(z-v) \Lambda\left(\frac{P_{\gamma \lambda} A(z)+\lambda-A(v)}{\|z-v\|}\right) f(v) d v, \quad z \in \mathbb{R}^{n},
$$

and $P_{\gamma \lambda}$ is defined as follows. Let $P \in C_{0}^{\infty}\left(B_{1}(0)\right)$ be an even function with $\int_{\mathbb{R}^{n}} P_{\lambda}(z) d z \equiv 1$, where as usual $P_{\lambda}(z) \equiv \lambda^{-d} P\left(\lambda^{-\alpha} z\right)$, and let $f \longrightarrow P_{\lambda} f$ be the convolution operator whose kernel is $P_{\lambda}(z)$. We choose $\gamma$ to be a small, fixed number, depending only on $\|A\|_{\text {comm }}$, such that

$$
\left|\frac{\partial}{\partial \lambda} P_{\gamma \lambda} A(z)\right| \leq \frac{1}{2} .
$$

Next let $g \in C_{0}^{\infty}\left(\mathbb{R}^{n}\right)$ with $\|g\|_{2,1 / \omega}=1$ and observe that

$$
\left\|\mathbb{D}_{n} S f\right\|_{2, \omega}=\sup \left|\int_{\mathbb{R}^{n}} \mathbb{D}_{n} S f g d z\right|,
$$

where the supremum is taken over all such $g$. Moreover,

$$
\begin{aligned}
-\int_{\mathbb{R}^{n}} \mathbb{D}_{n} S f g d z= & \int_{0}^{\infty} \int_{\mathbb{R}^{n}} \frac{\partial}{\partial \lambda}\left(\mathbb{D}_{n} S_{\lambda} f P_{\lambda} g\right) d z d \lambda \\
= & \int_{0}^{\infty} \int_{\mathbb{R}^{n}} \mathbb{D}_{n} \frac{\partial}{\partial \lambda} S_{\lambda} f P_{\lambda} g d z d \lambda \\
& +\int_{0}^{\infty} \int_{\mathbb{R}^{n}} \mathbb{D}_{n} S_{\lambda} f \frac{\partial}{\partial \lambda} P_{\lambda} g d z d \lambda \\
= & I+I I .
\end{aligned}
$$


We recall that we have defined a parabolic fractional derivative operator $\mathbb{D}$ by the Fourier multiplier

$$
\widehat{\mathbb{D} f} \equiv\|\zeta\| \widehat{f}
$$

We observe that $\partial P_{\lambda} / \partial \lambda=\mathbb{D} \widetilde{Q}_{\lambda}$ where $\widetilde{Q}_{\lambda}$ is an approximation to the zero operator $\left(i . e ., \widetilde{Q}_{\lambda} 1 \equiv 0\right)$ whose convolution kernel satisfies the standard kernel estimates (3.1) and (3.2). We leave the details of this routine estimate to the reader, noting only that to prove it, one uses the fact that the kernel of $\partial P_{\lambda} / \partial \lambda$ has not only mean value zero, but also vanishing first moments, since we have chosen $P(z)$ to be an even function. Thus since $\mathbb{D}_{n}=i \mathbb{D}^{-1}(\partial / \partial t)$, we have

$$
|I I|=\left|\int_{0}^{\infty} \int_{\mathbb{R}^{n}} \frac{\partial}{\partial t} S_{\lambda} f \widetilde{Q}_{\lambda} g d z d \lambda\right|
$$

Since $\|g\|_{2,1 / \omega}=1$, weighted Littlewood-Paley theory implies that

$$
\int_{0}^{\infty} \int_{\mathbb{R}^{n}}\left(\widetilde{Q}_{\lambda} g\right)^{2}\left(\frac{1}{\omega}\right) d z \frac{d \lambda}{\lambda} \leq c_{\omega}
$$

Hence, by Schwarz's inequality,

$$
|I I|^{2} \leq c_{\omega} \int_{0}^{\infty} \int_{\mathbb{R}^{n}}\left|\frac{\partial}{\partial t} S_{\lambda} f\right|^{2} \omega \lambda d z d \lambda
$$

Now let

$$
w\left(x_{0}, z\right) \equiv \int_{\mathbb{R}^{n}} J(z-v) \Lambda\left(\frac{x_{0}-A(v)}{\|z-v\|}\right) f(v) d v
$$

and define the Kenig-Stein mapping

$$
\rho(\lambda, z)=\left(\lambda+P_{\gamma \lambda} A(z), z\right) .
$$

Since $w \circ \rho(\lambda, z)=S_{\lambda} f(z)$, we have for $z=(x, t)$ that

$$
\begin{aligned}
\frac{\partial}{\partial t} S_{\lambda} f(z) & =\frac{\partial}{\partial t}(w \circ \rho)(\lambda, x, t) \\
& =w_{t} \circ \rho(\lambda, x, t)+w_{x_{0}} \circ \rho(\lambda, x, t) \frac{\partial}{\partial t} P_{\gamma \lambda} A(x, t)
\end{aligned}
$$


To handle the contribution of $w_{t} \circ \rho$ to the integral in (4.6) we use the change of variable

$$
\widetilde{\lambda} \equiv \lambda+P_{\gamma \lambda} A(z)-A(z)
$$

which defines a mapping $(\lambda, z) \longrightarrow(\widetilde{\lambda}, z)$ of $\mathbb{R}_{+}^{n+1}$ with Jacobian

$$
1+\frac{\partial}{\partial \lambda} P_{\gamma \lambda} A(z)=\eta(\lambda, z)
$$

Since $\left|(\partial / \partial \lambda) P_{\gamma \lambda} A(z)\right| \leq 1 / 2$ for $\gamma$ small enough depending only on $\|A\|_{\text {comm }}$, and $\lim _{\lambda \rightarrow 0} P_{\gamma \lambda} A=A$, we deduce first that $1 / 2 \leq \eta \leq 3 / 2$ and thereupon that the above mapping is $1-1$ and onto $\mathbb{R}_{+}^{n+1}$. Changing variables as in (4.9) we find that by Theorem 2.5 ,

$$
\int_{0}^{\infty} \int_{\mathbb{R}^{n}}\left(w_{t} \circ \rho\right)^{2} \omega \lambda d z d \lambda \leq c_{J, \omega}(1+\beta)^{2 N}\|f\|_{2, \omega}^{2}
$$

as desired.

To handle the contribution of the second term in (4.8) to the integral in (4.6), we claim that the non-tangential maximal function of

$$
w_{x_{0}} \circ \rho(\lambda, x, t)
$$

is bounded on $L_{\omega}^{2}$ with norm on the order of $\left(1+\|A\|_{\text {comm }}\right)^{N}$. Indeed, the operator

$$
f \longrightarrow w_{x_{0}} \circ \rho(0, x, t)
$$

is of the form

$$
T_{A} f(z) \equiv \mathrm{p} \cdot \mathrm{v} \cdot \int_{\mathbb{R}^{n}} K(z-v) F\left(\frac{A(z)-A(v)}{\|z-v\|}\right) f(v) d v
$$

where

$$
K\left(\delta x, \delta^{2} t\right) \equiv \delta^{-d} K(x, t),
$$

$K \in C^{m}\left(\mathbb{R}^{n} \backslash\{0\}\right)$, for some large $m, F \in C^{k}\left(\mathbb{R}^{1}\right)$, for some large $k$, and where the parity of $K(x, t)$ in the $x$ variable is opposite to that of $F$. It is essentially the conclusion of [H2, Theorem 1], that such operators are bounded on $L^{2}$, and hence on $L_{\omega}^{2}$, with norm on the order of $\left(1+\|A\|_{\text {comm }}\right)^{N}$. The claim now follows by applying a standard argument involving Cotlar's inequality for maximal singular integrals, 
to pass from the singular integral on the boundary to the non-tangential maximal function. Furthermore

$$
\left\|\frac{\partial}{\partial t} P_{\gamma \lambda} A(z)\right\|^{2} \lambda d \lambda d z
$$

is a parabolic Carleson measure with a norm no larger than $\left\|\mathbb{D}_{n} A\right\|_{*}^{2}$. The desired bound for (4.6) now follows by the usual properties of Carleson measures.

We now turn to $I$ in (4.4). We integrate by parts in the integral defining $I$ to get

$$
\begin{aligned}
-I= & \int_{0}^{\infty} \int_{\mathbb{R}^{n}} \frac{\partial^{2}}{\partial \lambda^{2}} S_{\lambda} f P_{\lambda} g \lambda d z d \lambda \\
& +\int_{0}^{\infty} \int_{\mathbb{R}^{n}} \frac{\partial}{\partial \lambda} S_{\lambda} f \frac{\partial}{\partial \lambda} P_{\lambda} g \lambda d z d \lambda \\
= & I_{1}+I_{2} .
\end{aligned}
$$

Arguing as in the proof of (4.6) we find

$$
\begin{aligned}
\left|I_{2}\right|^{2} & =\left|\int_{0}^{\infty} \int_{\mathbb{R}^{n}} \frac{\partial}{\partial t} \frac{\partial}{\partial \lambda} S_{\lambda} f \widetilde{Q}_{\lambda} g \lambda d z d \lambda\right|^{2} \\
& \leq c_{\omega} \int_{\mathbb{R}^{n}}\left|\frac{\partial^{2}}{\partial t \partial \lambda} S_{\lambda} f\right|^{2} \omega \lambda^{3} d z d \lambda .
\end{aligned}
$$

Again

$$
\begin{aligned}
\frac{\partial^{2}}{\partial t \partial \lambda} S_{\lambda} f= & \frac{\partial^{2}}{\partial t \partial \lambda} w \circ \rho \\
= & \frac{\partial}{\partial t}\left(\left(w_{x_{0}} \circ \rho\right)\left(1+\frac{\partial}{\partial \lambda} P_{\gamma \lambda} A\right)\right) \\
= & \left(w_{x_{0} t} \circ \rho\right)\left(1+\frac{\partial}{\partial \lambda} P_{\gamma \lambda} A\right) \\
& +\left(w_{x_{0} x_{0}} \circ \rho\right)\left(\frac{\partial}{\partial t} P_{\gamma \lambda} A\right)\left(1+\frac{\partial}{\partial \lambda} P_{\gamma \lambda} A\right) \\
& +\left(w_{x_{0}} \circ \rho\right)\left(\frac{\partial^{2}}{\partial t \partial \lambda} P_{\gamma \lambda} A\right) \\
= & \Lambda_{1}+\Lambda_{2}+\Lambda_{3} .
\end{aligned}
$$


Since $\left|(\partial / \partial \lambda) P_{\gamma \lambda} A\right| \leq 1 / 2$, we have $\Lambda_{1} \leq 2\left|w_{x_{0} t} \circ \rho\right|$. We now use the change of variable (4.9), and invoke Theorem 2.13, to handle the contribution of $\Lambda_{1}$. As for $\Lambda_{2}$, since

$$
\left|\frac{\partial}{\partial t} P_{\gamma \lambda} A\right| \leq c(1+\beta)^{2} \lambda^{-1}
$$

we can use Theorem 2.5 to handle $w_{x_{0} x_{0}}$ in the same way that we treated $w_{t}$ above. Finally, we may handle the contribution of $\Lambda_{3}$, by the usual nontangential maximum-Carleson measure arguments, i.e., by exactly the same method that we used previously to treat the contribution of the second term on the right hand side of (4.8). Altogether, we obtain the desired bound for the term $I_{2}$.

It remains to estimate $I_{1}$. We note that $\lambda \mathbb{D}_{n} P_{\lambda} \equiv \widetilde{\widetilde{Q}}_{\lambda}$ where $\widetilde{\widetilde{Q}}_{\lambda}$ is an approximation to the zero operator whose kernel satisfies (3.1) and (3.2). Thus arguing as in the proof of (4.6), we obtain

$$
\begin{aligned}
\left|I_{1}\right| & =\left|\int_{0}^{\infty} \int_{\mathbb{R}^{n}} \frac{\partial^{2}}{\partial \lambda^{2}} S_{\lambda} f \widetilde{\widetilde{Q}}_{\lambda} g d z d \lambda\right| \\
& \leq c_{\omega}\left(\int_{0}^{\infty} \int_{\mathbb{R}^{n}}\left|\frac{\partial^{2}}{\partial \lambda^{2}} S_{\lambda} f\right|^{2} \omega \lambda d z d \lambda\right)^{1 / 2} .
\end{aligned}
$$

But

$$
\frac{\partial^{2}}{\partial \lambda^{2}} S_{\lambda} f=\left(w_{x_{0} x_{0}} \circ \rho\right)\left(1+\frac{\partial}{\partial \lambda} P_{\gamma \lambda} A\right)^{2}+\left(w_{x_{0}} \circ \rho\right)\left(\frac{\partial^{2}}{\partial \lambda^{2}} P_{\gamma \lambda} A\right)
$$

and these terms can each be handled by our earlier arguments. This concludes the proof of Theorem 2.16 for $S=S_{A}$. The proof for the second class of operators, $U_{A, B}$, is similar, and we leave the details to the interested reader.

\section{References.}

[Ca1] Calderón, A. P., Commutators of singular integral operators. Proc. Nat. Acad. Sci. USA 53 (1965), 1092-1099.

[Ca2] Calderón, A. P., Cauchy integrals on Lipschitz curves. Proc. Nat. Acad. Sci. USA 74 (1977), 1324-1327.

[Ch] Christ, M., Lectures on singular integral operators. CBMS regional conference series $\mathbf{7 7}$ Amer.Math. Soc., 1990. 
[CJ] Christ, M., Journé, J. L., Polynomial growth estimates for multilinear singular integral operators. Acta Math. 159 (1987), 51-80.

[CDM] Coifman, R., David, G., Meyer, Y., La solution des conjectures de Calderón. Adv. in Math. 48 (1983), 144-148.

[CMM] Coifman, R., MacIntosh, A., Meyer, Y., L'integrale de Cauchy definit un operateur borné sur $L^{2}$ sur les courbes Lipschitziennes. Ann. of Math. 116 (1982), 361-388.

[CW] Coifman, R., Weiss, G., Analyse harmonique non-commutatiave sur certains espaces homogènes. Lecture Notes in Math. 242 Springer-Verlag, 1971.

[CM] Coifman, R., Meyer, Y., Non-linear harmonic analysis, operator theory, and pde. Beijing Lectures in Harmonic Analysis, E. M. Stein, Ed. Princeton Univ. Press, 1986.

[D] Dahlberg, B., Poisson semigroups and singular integrals. Proc. Amer. Math. Soc. 97 (1986), 41-48.

[DKPV] Dahlberg, B., Kenig, C., Pipher, J., Verchota, G., Area integral estimates for higher order operators and systems. Ann. Inst. Fourier $\mathbf{4 7}$ (1997), 1425-1461.

[DJS] David, G., Journé, J. L., Semmes, S., Calderón - Zygmund operators, para-accretive functions, and interpolation. English language preprint of Opérateurs de Calderón - Zygmund. Revista Mat. Iberoamericana 1 (1985), 1-56.

[Do] Dorronsoro, J., A characterization of potential spaces. Proc. Amer. Math. Soc. 95 (1985), 21-31.

[Fng] Fang, X., Ph.D. Thesis, Yale University, 1990.

[FR] Fabes, E. B., Riviere, N. M., Symbolic Calculus of Kernels with Mixed Homogeneity. Singular Integrals. A. P. Calderón, Ed. Proc. Symp. Pure Math. Amer. Math. Soc. 10 (1967), 106-127.

[GR] Garcia-Cuerva, J., Rubio de Francia, J. L., Weighted Norm Inequalities and Related Topics. Math. Studies 116 North-Holland, 1985.

[H1] Hofmann, S., A characterization of commutators of parabolic singular integrals. In Fourier Analysis and Partial Differential Equations (Proceedings of the Conference in Harmonic Analysis and PDE, held in Miraflores de la Sierra, Spain, in 1992). Studies in Advanced Math., CRC, 1995, 195-210.

[H2] Hofmann, S., Parabolic singular integrals of Calderón type, rough operators, and caloric layer potentials. Duke Math J. 90 (1997), 209-259.

[HL] Hofmann, S., Lewis, J. L., Ann. Math. 144 (1996), 349-420.

[JnsP] Jones, P., Square functions, Cauchy integrals, analytic capacity, and harmonic measure, Harmonic Analysis and Partial Differential Equations. 
(J. Garcia-Cuerva, ed.) Lecture Notes in Math. 1384 (1989), 24-68, Springer-Verlag.

[LM] Lewis, J., Murray, M. A., On the Method of Layer Potentials for the Heat Equation in Time-varying Domains. Memoir of the Amer. Math. Soc. 114 (1995), 1-157.

[LiMS] Li, C., McIntosh, A., Semmes, S., Convolution singular integrals on Lipschitz surfaces. J. Amer. Math. Soc. 5 (1992), 455-481.

[MMT] Mitrea, D., Mitrea, M., Taylor, M., Layer Potentials, the Hodge Laplacian, and global boundary problems in nonsmooth Riemannian manifolds. Mem. Amer. Math. Soc. 150 (2001).

[Stz] Strichartz, R. S., Bounded mean oscillation and Sobolev spaces. Indiana Univ. Math. J. 29 (1980), 539-558.

Recibido: 5 de febrero de 1.999

Revisado: 24 de enero de 2.000

Steve Hofmann*

Department of Mathematics

University of Missouri Columbia, MO 65211-0001, U.S.A. hofmann@math.missouri.edu

and

John L. Lewis* Department of Mathematics

University of Kentucky Lexington, KY 40506-0027, U.S.A. john@ms . uky . edu

\footnotetext{
* Supported by an NSF Grant
} 